\title{
Neural mirroring during the observation of live and video actions in infants
}

Lieselot Ruysschaert ${ }^{1}$, Petra Warreyn ${ }^{1}$, Jan R. Wiersema ${ }^{1}$, Baris Metin, \& Herbert Roeyers ${ }^{1}$

${ }^{1}$ Department of Experimental Clinical and Health Psychology, Ghent University,

H. Dunantlaan 2, B-9000 Ghent, Belgium

Address correspondence to:

Lieselot Ruysschaert, Department of Experimental Clinical and Health Psychology, Ghent

University, Henri Dunantlaan 2, B-9000 Ghent, Belgium

Tel: +32 92649414

Fax: +32 92646489

E-mail: Lieselot.Ruysschaert@UGent.be / Lieselot.Ruysschaert@telenet.be

\section{Acknowledgements}

This article was written as a part of the first author's doctoral dissertation.

This research was supported by the Ghent University Research Fund (BOF) and the Marguerite-Marie Delacroix Fund.

We thank the participating day-care centres and organizations, all the participating children and their parents.

The procedure used in the current study was approved by the ethical committee of Ghent University. Informed consent was obtained from all subjects.

The authors declare that they have no conflict of interest.

Keywords : neural mirroring, mu suppression, video, infants, EEG, imitation 


\section{Highlights:}

- We measured significant EEG mu suppression during the observation and imitation of live goal-directed and mimicked actions in 18- to 36-months olds.

- In the video setting, where the goal-directed and mimicked actions were presented on video, no mu suppression was found during the observation conditions and less mu suppression was found during the action imitation condition compared to the live setting.

- The findings indicate the use of live actions in the design of paradigms investigating neural mirroring in infants. 


\section{Abstract}

Objective: Previous infant studies investigated neural mirroring during the observation of live or video actions. However, both methods have their (dis)advantages and studies using one of these methods are not always directly comparable. Therefore, the present study directly compared neural mirroring activity in a video setting with a live setting in infants between 18 and 36 months old.

Methods: Central mu rhythm suppression was measured through EEG recordings during the observation and imitation of the same goal-directed and mimicked actions presented either on video or live.

Results: Results revealed significant mu suppression during action imitation in both settings but stronger mu suppression was observed in the live setting during this condition. Significant mu suppression during the observation of goal-directed actions and mimicked actions was only observed in the live setting.

Conclusion: This study revealed a different influence of video and live actions on neural mirroring activity in infants.

Significance: It is recommended to use live actions to investigate neural mirroring in young children. 


\section{Introduction}

Mirror neurons, discovered in the macaque brain, are active both during execution and observation of actions (Di Pellegrino et al., 1992). Activity in this action observation/execution matching system has been measured by using the mu rhythm derived from electroencephalography (EEG) recordings (Arnstein et al., 2011). In adults, the power of the mu band oscillations, typically recorded from the sensorimotor cortex is suppressed both during action execution and observation (Gastaut et al., 1954; Muthukumaraswamy et al., 2004). Research findings suggest that a mu rhythm similar as in adults with an identical topography but at a lower frequency range can be recorded in children (Cochin et al., 2001).

Previous infant studies investigated neural mirroring during the observation of live (e.g., Nyström et al., 2010; Southgate et al., 2009) or video actions (e.g., Nyström, 2008; van Elk et al., 2008). The majority of the researchers used live stimuli reasoning that live stimuli provide a more realistic view on brain processing because these stimuli are efficiently processed due to their similarity with the real world (Carver et al., 2006). However, investigating neural mirroring in a live setting is often hampered by motor movement and/or vocalization of the participant which can increase the presence of artifacts in the brain imaging data (Junghofer et al., 2000). Some arguments can be postulated why it can be useful to use video stimuli in infant studies concerning neural mirroring. Firstly, a methodological advantage of video stimuli is the identical manner of presenting stimuli which makes it a direct and repeatable research setting (Barr et al., 2007). Furthermore, video presentation makes it possible to control more for motor planning or inhibited reaching which often occur in live observation studies (Järveläinen et al., 2001). Finally, as Nyström (2008) discussed, adult research often uses video stimuli to investigate neural mirroring (e.g., Oberman et al., 2005) which makes it interesting to compare these results with infant studies. However, it is suggested that infants do not process virtual 2D-video stimuli in the same way as real 3D-live 
stimuli because they do not always seem to understand the relationship between these two different kind of stimuli (Carver et al., 2006). These findings lead to the hypothesis that neural mirroring activity will be less pronounced using video stimuli (Shimada and Hiraki, 2006). Therefore, investigating neural mirroring responses to video compared to live stimuli in young infants seems interesting to learn more about the sensitivity of the infant neural mirroring.

To our knowledge, no studies so far have been conducted that directly compare infants neural mirroring responses to video and live stimuli. Therefore, the present study aimed to investigate infants' EEG mu wave suppression during the observation and execution of goaldirected and mimicked video actions compared to the presentation of the identical actions in a live setting in infants between 18 and 36 months old. Given the findings of Shimada and Hiraki (2006), who found stronger mu suppression during live actions in infants, we may expect to find differences in neural mirroring activity in video versus live conditions.

\section{Materials and methods}

\subsection{Participants}

The initial sample consisted of 68 infants who were allocated to either the live or the video setting. Prior to analyses, 34 tested infants were excluded due to insufficient artifact free data (video: $n=15$; live: $n=13$ ), insufficient or no cooperation of the infant (video: $n=$ 2; live: $n=2$ ) or technical problems with the EEG equipment or recording (live: $n=2$ ) which resulted in an inability to obtain clear EEG data for these infants. The final sample consisted of 34 infants ( 15 boys and 19 girls), between 18 and 36 months old $(M=26.44 ; S D=3.96$ ) with 16 participants in the video setting and 18 participants in the live setting. Both groups did not significantly differ on chronological age and gender; $F(1,32)=2.40, p=.131$ for age and $\chi^{2}(1)=0.54 ; p=.464$ for gender. All infants were healthy and developing normally. They 
were recruited through Flemish day-care centres and magazine or website advertisements. Characteristics of the participants are presented in Table 1. Handedness was determined by parent report or by analysing the video-recordings of the experiment.

\section{INSERT TABLE 1 ABOUT HERE}

\subsection{General procedure}

The experiment took place in a quiet laboratory room at Ghent University after obtaining parental informed consent. The EEG data were collected when the infant was alert and attending to the stimuli. In order to let the child get used to the environment and experimenter, the experiment started with a short free play moment with some attractive toys. Experimenter 1 (also the demonstrator of the actions during test phase) played with the child, while experimenter 2 prepared the appropriate EEG cap. Meanwhile, the procedure was explained to the parent. When the child was feeling comfortable, the parent was asked to sit at the table together with the child. Subsequently, the experimenters placed the EEG cap on the child's head while the child was watching a popular cartoon movie. Electrolytic conducting gel was applied with a syringe at each active electrode on the EEG-cap. During testing, each infant was seated on his/her caregiver's lap who was instructed to minimize interaction with the child. In the live setting, experimenter 1 sat at the other side of the table facing the child. In the video setting, a computer monitor was put on the table in front of the child. In both settings, the same stimuli were presented either live or on video at a viewing distance of approximately $60 \mathrm{~cm}$. White curtains surrounded the testing area to minimize distracting environmental influences; a white screen was placed around the infant and a white dropcurtain moved up and down between the different conditions (in the live setting). Two video cameras recorded the whole experiment; one focusing on experimenter 1 in the live setting or 
the monitor in the video setting and the other on the side profile of the child in both settings. Participants' behaviour (attention i.e. looking at the presentation of the video or live stimuli, vocalization, motor movement and imitation) was coded offline on the basis of these video recordings.

EEG data were collected during 4 experimental conditions with 5 different objects (i.e., a hippopotamus soft-toy, an egg-cup, a Pinocchio-like puppet, a car and a frog loupe) which were presented live during the live setting. (1) The experiment always started with the observation of a moving object in a non-goal-directed manner, dangling on a rope with the experimenter hidden behind a curtain out of the infant's view. Because the infant had no prior experience with the objects, this object observation condition was used as a baseline condition to which every subsequent condition was compared. (2) During the action observation condition, the participant observed the experimenter performing a simple goal-directed action with each object and a white box (for example, car is picked up, driven on top of the box, and is released at the edge of the box, so it falls (carefully) down at the other side of the box). These actions were 'goal-directed' because the object had a clear end position. Before demonstration, the experimenter asked the attention of the infant by saying 'look' and making eye-contact with the infant. Each action was demonstrated three times from the left side of the box and three times from the right side. The starting hand was counterbalanced between the different objects. (3) After observing the demonstrated actions, the infant was encouraged (non-)verbally in a non-specific way to imitate the observed action during the action imitation condition. Moreover, the experimenter asked the participant to imitate the observed actions by saying "your turn". Furthermore, the infant was given as much time as needed to perform the actions and it was possible that the experimenter repeated the action once more if it was not clear for the infant how to perform the actions. (4) During the fourth condition, the experimenter demonstrated hand movements identical to those used during the action 
observation condition but now without the object (i.e., mimicked actions). During this hand movement condition, the hand movements were executed without direct reference or gaze towards the participant which made the condition less social. The subject was expected to observe these actions but not to imitate them. Each hand movement was also demonstrated three times with the left hand and three times with the right hand.

During the video setting, the live actions of the object observation, action observation and hand movement conditions were replaced by the same actions but pre-recorded on video. The imitation condition was always live, regardless of the setting as the participants were always asked to imitate the observed actions which were either presented live or on video. The experiment always started with the object observation condition (baseline condition) in both settings. Afterwards, the action observation, action imitation and hand movement condition were presented for each object. The order in which these three conditions were presented to the participants was randomized across subjects, with the requirement that the action observation condition always preceded the action imitation condition. The five objects were always presented in the same order. Each demonstrated action (object movement, action observation and hand movement) lasted about (live setting) and exactly (video setting) 30 seconds per object. During action imitation, participants were given as much time as needed to perform the actions. The entire experiment lasted about 20 minutes. Afterwards, parents were debriefed and received a reward/gift card for participation.

\subsection{EEG data acquisition}

Brain Vision Recorder (Brain Products, 2007) was used to record electrical brain activity to an average reference from 32 active $\mathrm{Ag} / \mathrm{AgCl}$ electrodes through an EEG-amplifier (QuickAmp) with a sample rate of $500 \mathrm{~Hz}$. EEG data were recorded with $1 \mathrm{~s}$ time constant, a low pass filter of $70 \mathrm{~Hz}$ and a notch filter of $50 \mathrm{~Hz}$. Electrodes were placed according to the 
international 10-20 system (Jasper, 1958) embedded in a child-friendly stretch EEG-cap with a ground electrode placed at AFz (Easycap, Brain Products, GmbH, Munich, Germany). Electro-oculogram (EOG), both vertical and horizontal, were recorded by 3 additional electrodes. Horizontal EOG electrodes (HEOG) were applied next to the eyes, at the outer canthi. A vertical electro-oculogram (VEOG) was performed with an electrode above the eye, at position Fp2, compared with the common reference. Initially, we used an electrode positioned below the left eye for monitoring the vertical eye movement but many infants did not tolerate this electrode. However, in comparison with the data including these electrode, results showed no significant difference concerning the use of the common reference. An inter-electrode impedance of all electrodes at or below $10 \mathrm{k} \Omega$ was considered acceptable. Synchronization of the EEG signal with both camera recordings was done by pushing a button before the start of each condition. This button sent a marker signal to the EEG recording and simultaneously emitted a LED light signal visible on both cameras. The time intervals between the markers on these 2 recording systems were compared afterwards which allowed synchronization of the EEG data with the video recordings of the child's behaviour.

\subsection{Offline behaviour coding}

The behaviours on the camera recordings were coded offline with The Observer XT 9.0. (Noldus Information Technology, 2009) by ascribing start and stop codes to the child's attentive behaviour, vocalizations, motor movements, imitation behaviour and the different experimental conditions. The fragments where the child was sitting still and quietly observed the demonstrations (during the object movement, hand movement and action observation condition) or was actually imitating (during the imitation condition) were used for further analysis. Intervals with excessive motor movements and vocalizations were excluded beforehand to minimize contamination of the EEG signal. In addition, artifact rejection was 
performed in the subsequent Brain Vision Analyzer analyses to control for artifacts. This insured that differences in terms of mu suppression between conditions could not be explained by overall differences between conditions in motor and vocalization behaviour of the infant.

One observer, who was blind for the setting in which the child was tested, coded quality of imitation of the participants during the action imitation condition. The coding was based on three different criteria per object. For example, for driving the car, it was coded if (1) the child drove the car on the side of the box, (2) followed by driving on top of the box and (3) finally dropped it at the other side of the box. Score 1 was given for every criteria the infant met. Afterwards, a quality of imitation score was calculated by taking the mean of the best scores for each object with a maximum of 3 per object. In this sample, participants in the video setting obtained a mean score of $1.80(S D=.43)$ and a mean score of $2.04(S D=.38)$ for the live setting, indicating that overall the imitation performance of the infants met (almost) 2 of the 3 criteria in both settings. An independent coder double-coded $25 \%$ randomly chosen videos to assess inter-observer reliability. An excellent level of reliability was achieved with a Cronbach’s Alpha Coefficient of .94 (Cronbach, 1951).

\subsection{EEG data processing}

We used Brain Vision Analyzer (Brain Products, 2007) for offline analyses of the recorded EEG data. Data recorded from electrodes C3 and C4 were further investigated because mu rhythm is defined as oscillations measured over the sensorimotor cortex (Marshall et al., 2011; Muthukumaraswamy et al., 2004). The recorded EEG data were first inspected visually offline to eliminate bad recordings. In addition, bad channels were excluded before re-referencing to prevent spreading of bad data. The remaining channels were re-referenced to an average reference. Afterwards, the EEG-signal was filtered with a high pass filter of $0.1 \mathrm{~Hz}$, a low pass filter of $30 \mathrm{~Hz}$, and a $50 \mathrm{~Hz}$ notch filter. Subsequently, the 
EEG data were corrected for horizontal and vertical eye movements using the Gratton and Coles algorithm (Gratton et al., 1983). The remaining EEG data were further segmented to separate data per experimental condition and afterwards divided in 1-s epochs with $50 \%$ overlap. Bad segments were removed with artifact rejection using a maximal allowed voltage step of $100 \mu \mathrm{V}$ per sampling point; a maximal allowed absolute difference of $400 \mu \mathrm{V}$ between two values in the segment and an activity of $0 \mu \mathrm{V}$ during maximum 100 milliseconds. In this way, an average of 229.24 segments $(S D=86.00)$ remained. Fast Fourier Transforms (FFTs), with a Hanning window of $10 \%$, were performed on the remaining segments and the resulting magnitudes were averaged for each condition. Similar as in analogous experiments (e.g., Lepage and Théoret, 2006; Muthukumaraswamy et al., 2004), the individual mu rhythm bandwidths were conducted by subtracting the baseline condition from the imitation condition for each subject individually. In this way, we controlled for the differences in spectral power that could be caused by mere presentation of visual stimuli. In addition, the $3-\mathrm{Hz}$ interval around the maximal power difference of this subtraction over the central electrodes was calculated. This procedure was selected because it enables the precise definition of the frequency band that is modulated by the execution of actions in each individual subject. Contrasting the baseline and the imitation condition results in a clearer individual mu rhythm (Muthukumaraswamy et al., 2004). The mean peak in the live group was $8.1 \mathrm{~Hz}(S D=0.75)$ and $8.1 \mathrm{~Hz}(S D=0.60)$ for the video group. This is in agreement with previous studies on mu/alpha rhythm frequencies in infants (Marshall et al., 2002; Stroganova et al., 1999).

Following the procedure used by Oberman and colleagues (2005) and Raymaekers and colleagues (2009), mu wave suppression was calculated as a ratio of the mu wave power in the different conditions. Specifically, we calculated the individual mu power during the experimental conditions (the action observation, action imitation and hand movement condition) relative to the mu power in the baseline condition (the object movement condition). 
A ratio was used to control for the individual variability in absolute EEG power due to individual differences such as electrode impedance or scalp thickness. Given the non-normal distribution of ratio data, a log transform was computed for each ratio. A negative value indicates mu suppression, a positive value represents mu augmentation, and a zero value indicates no mu suppression, as compared to the baseline.

\section{Results}

Counterbalancing of the order of the presented conditions (the action observation condition/action imitation condition versus the hand movement condition) in both settings (live setting and video setting) had no effect on the mu suppression as measured at the central (C3 and C4) electrode positions, all $-.47<t(16)<1.33$, all $p>.05$ and all $-.67<t(14)<.29$, all $p>.05$ respectively. Therefore, the order of presentation of the conditions was not further included as a factor in the analyses.

A $3 \times 2 \times 2$ repeated-measures ANOVA was conducted with condition (hand movement observation, action observation and action imitation) and hemisphere (C3 and $\mathrm{C} 4)$ as withinsubjects factors and setting (live setting vs. video setting) as between-subjects factor. Results revealed a significant main effect of setting with more mu suppression in the live setting $(M=$ $-.23, S D=.24)$ compared to the video setting $(M=-.03, S D=.17) ; F(1,32)=7.75, p=.009$ and a significant main effect of condition, $F(2,31)=7.56, p=.002$. Follow-up contrasts demonstrated significantly more mu suppression during the action imitation condition $(M=$ $-.31, S D=.44)$ compared to the hand movement condition $(M=-.09, S D=.23)$ and the action observation condition $(M=-.01, S D=.16)$ with $F(1,32)=12.41, p=.001$ and $F(1,32)=$ 15.60, $p<.001$ respectively, and significantly stronger mu suppression during hand 
movement observation compared to action observation, $F(1,32)=5.17, p=.030$. No main effect of hemisphere was found with $F(1,32)=.24, p=.627$. Furthermore, no significant 2and 3-way interactions were found (all $p>.05)$.

In addition to investigating the quantitative differences between live and video presentation, we also tested whether or not there was significant mu suppression in both presentation modes separately. Therefore, we conducted separate t-tests to see if the mean values per condition per presentation mode at electrode positions $\mathrm{C} 3$ and $\mathrm{C} 4$ significantly differed from zero.

Results in the live setting revealed significant mu suppression at electrode position $\mathrm{C} 3$ during the hand movement condition, the action observation condition and the action imitation condition, $t(17)=-3.03, p=.008 ; t(17)=-3.45, p=.003$ and $t(17)=-2.83, p=.012$ respectively. $\mathrm{Mu}$ suppression at electrode position $\mathrm{C} 4$ was significant during the hand movement condition, $t(17)=-2.29, p=.035$ and the action imitation condition, $t(17)=-3.46$, $p=.003$ but not during the action observation condition, $t(17)=-.52, p=.607$.

For the video setting, mu enhancement at electrode position C3 was significantly different from zero during the hand movement condition and the action observation with $t(15)$ $=2.16, p=.047$ and $t(15)=2.83, p=.013$ respectively. Mu suppression tended to differ significantly from zero during the action imitation condition, $t(15)=-1.99, p=.065$. No mu suppression or enhancement was found during both observation conditions at electrode $\mathrm{C} 4$ with $t(15)=0.02, p=.985$ for the hand movement condition and $t(15)=0.62, p=.547$ for the action observation condition. Only mu suppression at electrode position $\mathrm{C} 4$ during the action imitation was significant with $t(15)=-2.44, p=.027$. Figure 1 shows the mean mu power values for both settings at $\mathrm{C} 3$ and $\mathrm{C} 4$ separately during each condition.

INSERT Figure 1 ABOUT HERE 
In order to ensure that the differences between the live and the video setting are not secondary to differences in topography, we also analyzed data at frontal (i.e., average of F3 and F4) and parietal (i.e., average of P3 and P4) electrode positions.

In the live setting, mean mu suppression at the frontal electrode sites F3 and F4 differed significantly from zero during the hand movement condition $(M=-.11, S D=.18)$, the action observation condition $(M=-.09, S D=.17)$ and the action imitation condition $(M=$ $-.19, S D=.32)$, with $t(17)=-2.57, p=.020 ; t(17)=-2.17, p=.044$ and $t(17)=-2.44, p=.026$ respectively. In the video setting, frontal mu suppression was only significant during the action imitation condition, with $t(15)=-2.30, \mathrm{p}=.036$ but not during the hand movement condition $(M=-.03, S D=.15)$ and the action observation condition $(M=.03, S D=.22)$, with $t(15)=-.72, p=.484$ and $t(15)=.59, p=.567$ respectively.

Parietal mu suppression was significant during the live action observation $(M=-.17$, $S D=.15)$ and the live action imitation condition $(M=-.15, S D=.28)$, with $t(17)=-4.90, p<$ .001 and $t(17)=-2.31, p=.034$ respectively but not during the live hand movement condition $(M=-.10, S D=.24), t(17)=-1.7, p=.112$. No significant parietal mu suppression was found in the video group during the hand movement condition $(M=-.01, S D=.20)$, the action observation $(M=.02, S D=.19)$ and the action imitation condition $(M=-.02, S D=.22)$, with $t(15)=-.28, p=.782, t(15)=.34, p=.740$ and $t(15)=-.40, p=.697$ respectively. Figure 2 demonstrates the mean mu power values for both settings at frontal and parietal electrode positions during each condition.

Additional analyses in the live group revealed only a significant effect of location during the action imitation condition, with more central mu suppression compared to frontal and parietal mu suppression, $t(17)=2.17, p=.044$ and $t(17)=-3.04, p=.007$. 
Additionally, it was evaluated whether motor activity during the observation of actions and hand movements, quality of imitation during the action imitation condition and attentive behaviour during both observation conditions differed between the two settings. Results revealed no significant differences regarding motor activity between the live and the video setting, with all $t(31)<1.7$, all $p>.05$. The live group tended to imitate better than the video group with $t(32)=1.75, p=.090$. The infants in the video setting spent more time looking at the presented stimuli than the infants in the live setting with $t(31)=-5.48, p<.001$.

Finally, activity recorded from an occipital electrode $(\mathrm{Oz})$ was investigated to evaluate if the observed central suppression was related to the mu rhythm and not to other possibly overlapping activity such as posterior alpha activity. During action imitation in both settings, no significant suppression was found at electrode $\mathrm{Oz}$ in the frequency band under investigation, $t(33)=-1.09, p=.282$. When central mu suppression was compared to occipital activity, we found significant more central suppression $(M=-.31, S D=.44)$ compared to occipital suppression in the same frequency band $(M=-.07, S D=.37), t(33)=-2.39, p=.023$. These results indicate that the observed mu suppression was specific to the central electrode positions and was not the result of occipital activity.

\section{Discussion}

To our knowledge, this is the first study investigating neural mirroring during observation and imitation of video goal-directed and mimicked actions compared to the same actions presented live in infants. As Marshall and Meltzoff (2010) suggested, we included both observation and execution conditions.

As expected, given the motor properties of the mu wave (Lepage and Théoret, 2007), the infants in both settings showed significant mu suppression during the action imitation 
condition. However, only during the live presentation, the infants demonstrated significant mu suppression during both observation conditions. The finding of significant mu suppression during both observation and imitation in the live setting suggests the presence of a functional action observation/execution matching system in infants between 18 and 36 months old (Marshall and Meltzoff, 2010). The occurrence of mu wave suppression during the observation of live non-goal-directed hand movements, suggests that the observation of live motor movements alone is sufficient to provoke mu suppression in infants between 18 and 36 months old (Oberman et al., 2005). In contrast, the mere observation of video hand movements and goal-directed actions was insufficient to evoke mu suppression, which differed significantly with the live setting observation conditions.

The results of the current study suggest different neural mirroring activation during the observation of video and live actions in infants. Since the children in the present study paid more attention to the presentation of the video stimuli than to the live stimuli, less mu suppression in the video setting cannot be explained by different attentive behaviour dependent on the setting. Neither was neural mirroring activation in the live setting the result of motor activity during observation, since no significant differences were found between the two settings concerning motor activity during both observation conditions. In the present study, although the quality of imitation of the live presented actions was slightly better than the video actions, the difference was not significant, which suggests that infants understood the imitation tasks both when it was presented on video or live. This is in line with the findings of Barr and Hayne (1999) that infants from 18-months old onwards can imitate video modelled actions. Furthermore, the same tasks were used in the video and the live setting which excludes a potentially different impact of tasks on neural mirroring activation in both settings. Therefore, we can conclude that the infants responded neurologically different to the observation of video compared to live actions probably due to a different visual experience 
with 2D stimulus presentation in contrast to real 3D object presentation (Shimada and Hiraki, 2006). Additionally, research revealed that infants under 3 years of age find it difficult to symbolize 2D scale models as 3D real objects (DeLoache, 2000). Therefore, it could be suggested that less mu suppression in the video group is the result of inadequate visual processing. However, research revealed that visual processing and attention can be measured from the age of 6.5 months old using appropriate video paradigms (Jacobson et al., 1992). This suggests that visual, cognitive and neural processing can be reliably assessed in infancy during video stimulus presentation and that impaired visual information processing cannot fully explain the results of the present study.

In the live group, central mu suppression was significantly stronger compared to frontal and parietal electrode activity during the action imitation condition. Except for significant frontal mu suppression during the action imitation condition, no significant frontal and parietal mu suppression was found in the video group during all three conditions. Therefore, it can be concluded that the differences in central mu suppression between the live and the video group cannot be explained by differences in topography.

Finally, we found a significant effect of condition with significantly more mu suppression during the action imitation condition compared to the hand movement condition and the action observation condition, and significantly stronger mu suppression during hand movement observation compared to action observation. This was also observed in previous research (e.g., Lepage and Théoret, 2006), and is not surprising since children actually move during this condition, as opposed to the observation condition where they are required to sit still. While the mu suppression during the observation conditions is mainly caused by observing goal-directed actions, mu suppression during the action imitation condition is also caused by actual movement. Alternatively, the main effect of condition could be explained by the procedure that was used for calculating the individual mu rhythm bandwidths by 
subtracting the baseline condition (i.e., the object observation condition) from the imitation condition for each subject individually, with a mean peak in the live group of $8.1 \mathrm{~Hz}(S D=$ $0.75)$ and $8.1 \mathrm{~Hz}(S D=0.60)$ in the video group. This procedure was selected based on previous analogous experiments (e.g., Lepage and Théoret, 2006; Muthukumaraswamy et al., 2004). However, the frequencies of the maximum difference between the baseline and the action observation condition in our study was smaller in both settings, with a mean frequency of $7.08 \mathrm{~Hz}(S D=1.49)$ and $7.60 \mathrm{~Hz}(S D=1.66)$ for the video and live setting respectively. Comparing the baseline condition with the hand movement condition resulted in a maximum difference frequency of $7.63 \mathrm{~Hz}(S D=1.19)$ and $8.03 \mathrm{~Hz}(S D=1.14)$ for the video and the live setting respectively. Although these peak frequencies are somewhat lower than the peak frequencies we obtained when using the action imitation condition $(M=8.1, S D=0.75$ in the live condition and $M=8.1, S D=0.60$ in the video condition), they are still well within each child's individual 3Hz-frequency band. However, we cannot fully exclude the possibility that this small difference in peak frequency may have influenced the main effect of condition in our study.

Although the findings of the present study are in agreement with previous research (e.g., Järveläinen et al., 2001; Shimada and Hiraki, 2006), some limitations need to be considered. First, the differences between the two settings could be due to the variation in the duration of the live demonstrations in contrast to the pre-recorded video demonstrations. It seems inevitable that during live demonstrations, the experimenter unconsciously adapted the demonstration time to each individual participant, contingent upon its behaviour, whereas the duration of the pre-recorded video demonstrations was not dependent on the child's behaviour. However, it should be noted that in the present study, only the fragments where the child was attentive to the demonstrations during live as well as during video conditions were used for further analyses. Secondly, especially during the live setting, inhibited movement or 
motor planning can cause significant neural mirroring activity during the observation tasks. By excluding the fragments with too many motor movements and vocalizations beforehand during video coding and by using a profound artefact rejection during the EEG analyses, we tried to control for these artefacts. However, we could not control for all of it which makes it possible that this can differ between the two settings with different neural mirroring activity as result.

To our knowledge, this is the first study that directly compared neural mirroring activity during the observation of hand movements and goal-directed actions in a video setting with the same actions in a live setting in infants between 18 and 36 months old. Therefore, these findings need to be replicated in future studies with larger sample sizes.

To conclude, our study revealed less mu suppression during goal-directed action observation and hand movement observation when stimuli were shown on video in comparison with the observation of live actions in infants between 18 and 36 months old. These findings clearly indicate a different sensorimotor processing of video compared to live presented actions in infancy and imply the importance of using live actions to investigate neural mirroring activity in infancy. Apparently, live movements have a higher ecological validity than video actions. This result can be taken into account in the design of adequate paradigms to investigate neural mirroring activity in infancy. 


\section{References}

Arnstein D, Cui F, Keysers C, Maurits NM, Gazzola V. mu-Suppression during Action Observation and Execution Correlates with BOLD in Dorsal Premotor, Inferior Parietal, and SI Cortices. J Neurosci. 2011;31:14243-9.

Barr R, Hayne H. Developmental changes in imitation from television during infancy. Child Dev. 1999;70:1067-81.

Barr R, Muentener P, Garcia A, Fujimoto M, Chavez V. The effect of repetition on imitation from television during infancy. Dev Psychobiol. 2007;49:196-207.

Carver LJ, Meltzoff AN, Dawson G. Event-related potential (ERP) indices of infants' recognition of familiar and unfamiliar objects in two and three dimensions. Dev Sci. 2006;9:51-62.

Cochin S, Barthelemy C, Roux S, Martineau J. Electroencephalographic activity during perception of motion in childhood. Eur J Neurosci. 2001;13:1791-6.

Cronbach LJ. Coefficient alpha and the internal structure of tests. Psychometrika. 1951;16:297-334.

DeLoache JS. Dual representation and young children's use of scale models. Child Dev. 2000;71:329-38.

Di Pellegrino G, Fadiga, L., Fogassi, L., Gallese, V., Rizzolatti, G. Understanding motor events: A neuropsychological study. Exp Brain Res. 1992;91:176-80.

Gastaut H, Dongier, M., Courtois, G. On the significance of "wicket rhythms" ("rhythmes en arceau") in psychosomatic medicine. Electroencephalogr Clin Neurophysiol. 1954;6:687.

Gratton G, Coles, M.G.H., Donchin, E. A new method for off-line removal of ocular artifact. Electroencephalogr Clin Neurophysiol. 1983;55:468-84. 
Jacobson, S. W., Jacobson, J. L., Oneill, J. M., Padgett, R. J., Frankowski, J. J., Bihun, J. T. Visual expectation and dimensions of infant information-processing. Child Dev. 1992; 63:711-724.

Jarvelainen J, Schurmann M, Avikainen S, Hari R. Stronger reactivity of the human primary motor cortex during observation of live rather than video motor acts. Neuroreport. $2001 ; 12: 3493-5$.

Jasper HH. The ten-twenty electrode system of the International Federation. Electroencephalogr Clin Neurophysiol. 1958;10:370-5.

Junghofer M, Elbert T, Tucker DM, Rockstroh B. Statistical control of artifacts in dense array EEG/MEG studies. Psychophysiology. 2000;37:523-32.

Lepage JF, Theoret H. EEG evidence for the presence of an action observation-execution matching system in children. Eur J Neurosci. 2006;23:2505-10.

Lepage JF, Theoret H. The mirror neuron system: grasping others' actions from birth? Dev Sci. 2007;10:513-23.

Marshall PJ, Bar-Haim Y, Fox NA. Development of the EEG from 5 months to 4 years of age. Clin Neurophysiol. 2002;113:1199-208.

Marshall PJ, Meltzoff, A.N. Neural Mirroring Systems : Exploring the EEG mu rhythm in human infancy. Dev Cognit Neurosci. 2010;1:110-23.

Marshall PJ, Young T, Meltzoff AN. Neural correlates of action observation and execution in 14-month-old infants: an event-related EEG desynchronization study. Dev Sci. 2010;14:474-80.

Muthukumaraswamy SD, Johnson BW, McNair NA. Mu rhythm modulation during observation of an object-directed grasp. Cognit Brain Res. 2004;19:195-201.

Nystrom P. The infant mirror neuron system studied with high density EEG. Soc Neurosci. $2008 ; 3: 334-47$. 
Nystrom P, Ljunghammar T, Rosander $\mathrm{K}$, von Hofsten C. Using mu rhythm desynchronization to measure mirror neuron activity in infants. Dev Sci. 2010;14:32735.

Oberman LM, Hubbard EM, McCleery JP, Altschuler EL, Ramachandran VS, Pineda JA. EEG evidence for mirror neuron dysfunction in autism spectrum disorders. Cognit Brain Res. 2005;24:190-8.

Raymaekers R, Wiersema JR, Roeyers H. EEG study of the mirror neuron system in children with high functioning autism. Brain Res. 2009;1304:113-21.

Shimada S, Hiraki K. Infant's brain responses to live and televised action. Neuroimage. 2006;32:930-9.

Southgate V, Johnson MH, Osborne T, Csibra G. Predictive motor activation during action observation in human infants. Biol Lett. 2009;5:769-72.

Stroganova TA, Orekhova EV, Posikera IN. EEG alpha rhythm in infants. Clin Neurophysiol. 1999;110:997-1012.

van Elk M, van Schie HT, Hunnius S, Vesper C, Bekkering H. You'll never crawl alone: Neurophysiological evidence for experience-dependent motor resonance in infancy. Neuroimage. 2008;43:808-14. 


\section{Table 1. Subject characteristics}

\begin{tabular}{ccccc}
\hline & Live setting $(n=18)$ & & Video setting $(n=16)$ \\
\cline { 2 - 2 } $\begin{array}{l}\text { Chronological age } \\
\text { (months) }\end{array}$ & $25.47(4.35)$ & & $27.54(3.28)$ & $F(1,32)=2.40$ \\
Mean $(S D)$ & $19.80-35.30$ & & $20.20-30.70$ & \\
\hline Age Range & $9: 9$ & $6: 10$ & $\chi^{2}(1)=0.54$ \\
\hline Gender ratio M:F & $12: 5: 1$ & $12: 4$ & \\
\hline Handedness (R:L:ambi) & & & & \\
\hline
\end{tabular}




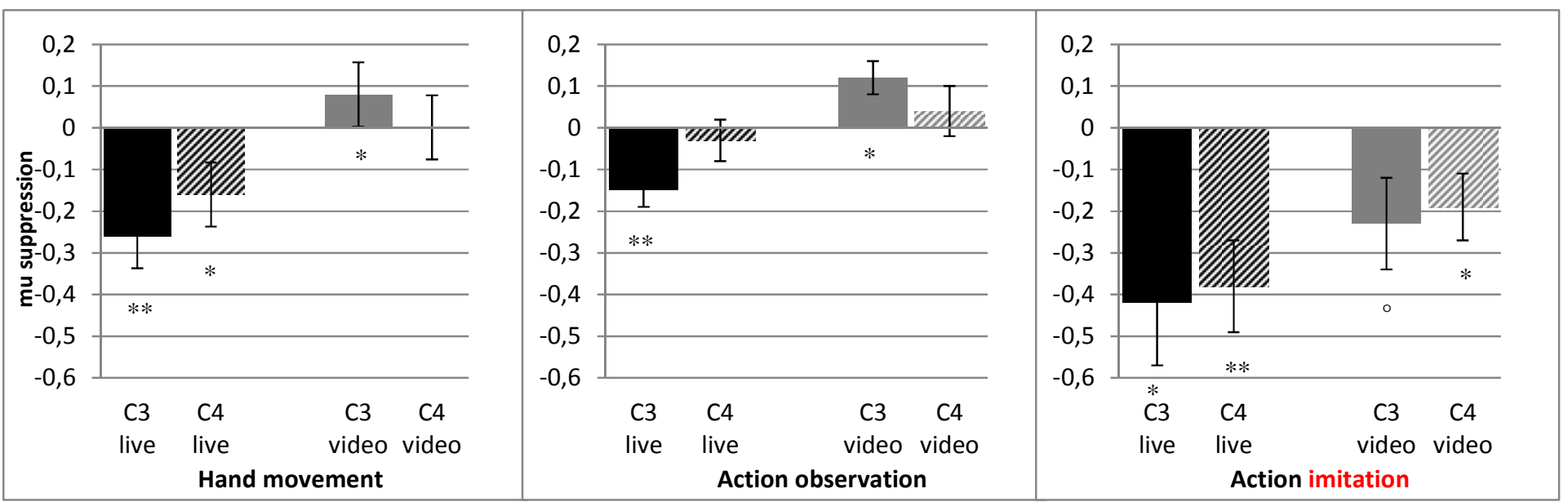

Figure 1. Mean mu power values for both settings during all three conditions at C3 and C4 separately with C3 live = mu suppression at electrode position $C 3$ during the live setting; $C 4$ live = mu suppression at electrode position C4 during the live setting; $C 3$ video $=m u$ suppression at electrode position $C 3$ during the video setting; $C 4$ video $=m u$ suppression at electrode position $C 4$ during the video setting. Error bars show \pm 1 standard error.

${ }^{\circ} p<.10 ; * p<.05 ; * * p<.01$. 


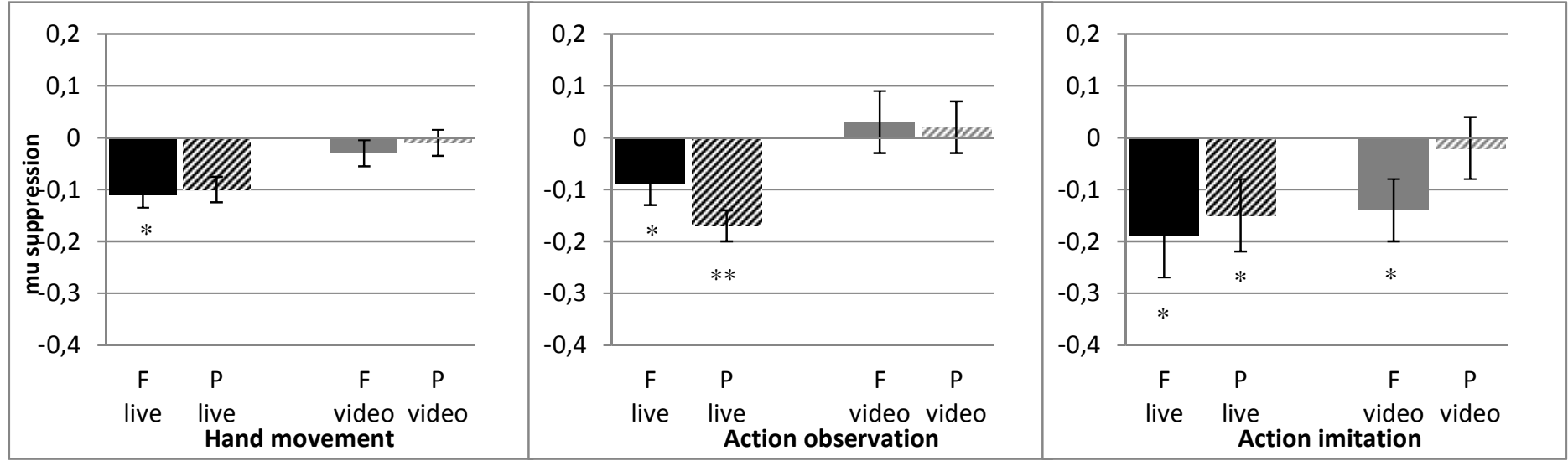

Figure 2. Mean mu power values for both settings during all three conditions at frontal (average of F3 and F4; F) and parietal (average of P3 and P4; P) electrode positions; live = live setting; video = video setting. Error bars show \pm 1 standard error.

$* p<.05 ; * *<<.001$. 